\title{
Large-eddy simulations for wind turbine blade: rotational augmentation and dynamic stall
}

\author{
Y. Kim, I.P. Castro, and Z.T. Xie
}

\section{Introduction}

Wind turbines operate in the atmospheric boundary layer and their rotating mechanisms provide complicated aerodynamic phenomena within their operating environments. When the upstream wind is uniform and normal to the plane of a rotating blade, rotational effects (i.e. rotational augmentation) emerge. Rotational augmentation means that stall occurs at a higher angle of attack on the rotating blade section than it does on an analogous stationary airfoil. It is conjectured that the rotational augmentation is a main reason for the deviations between the predicted and measured performance of wind turbines [11]. Understanding the mechanism for rotational augmentation is important to design prediction models.

At yaw, the blades operate in periodically oscillating condition and dynamic stall is dominant on the flow around the blade [10]. The generated force accumulates fatigue loads reducing the life cycle of wind turbines. Due to a strong 3-D phenomenon at a deep stall, it would be extremely challenging to predict the flow on the pitching airfoil by using Reynolds Averaged Navier-Stokes (RANS) approaches.

In this study, rotational augmentation and dynamic stall are investigated using large-eddy simulations (LES). In particular, the effect of freestream turbulence on the dynamic stall hysteresis is studied by using the recently developed divergencefree turbulence inflow generation method [6]. Up to our knowledge, there is very little works on the effect of freestream turbulence on the dynamic stall characteristics and none with LES approaches.

Y. Kim · I.P. Castro · Z.T. Xie

Faculty of Engineering and the Environment, University of Southampton. e-mail: \{yusik.kim, i.castro, Z.Xie\}@soton.ac.uk 

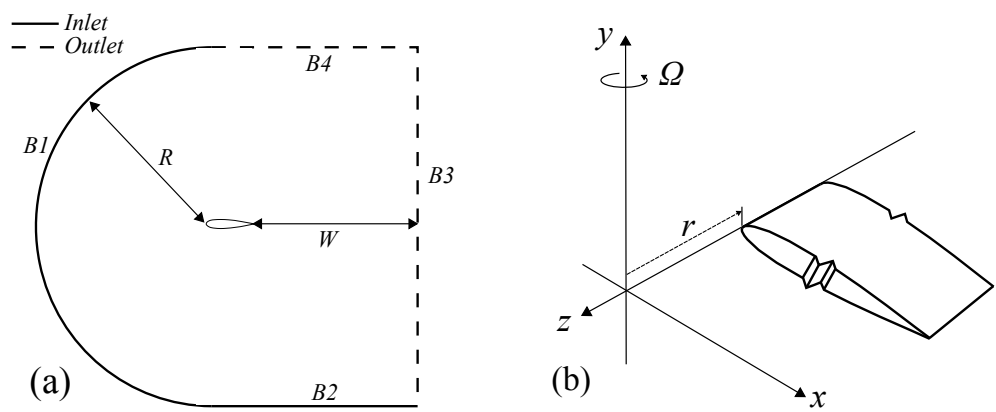

Fig. 1 Schematics of (a) the domain and boundary conditions (not to scale) and (b) coordinate system on the rotating blade.

\section{Methodology}

A NACA 0012 airfoil was used for both the rotational augmentation and dynamic stall studies. A rotating reference frame was applied for the former and a dynamic mesh was used for the latter. A common methodology for both studies is described first, then details will be explained in the following sections. A Reynolds number $R e=U_{\infty} c / v=135,000$ was used where $U_{\infty}$ is the freestream velocity and $c$ is the chord length. The domain size was $R=22 c, W=33 c$ (see Fig.1a). The mixed-timescale (MTS) SGS model [3] was used. A simple top-hat filter was applied for the explicit filter in the MTS model.

A second order, implicit scheme was used for the time derivative and the second order bounded Gamma scheme [4]) was used for the convection term. The finite volume based, transient incompressible flow solver from OpenFOAM [8] was used and the PIMPLE (SIMPLE + PISO) algorithm was used for the velocity-pressure coupling. Constant velocity components and zero pressure gradients were imposed on the inlet boundary and zero velocity gradients and a constant pressure were imposed on the outlet boundary. Note that the $x$ and $y$ coordinates (with the origin at the leading edge of the airfoil) used throughout all simulations represented the streamwise and cross-flow directions respectively.

\section{Rotational augmentation}

Gross et al.'s [2] method was applied for the rotational augmentation study. They derived the governing equations in a rotating reference frame by using an orderof-magnitude analysis for the blade sectional simulation with a periodic boundary condition in the spanwise direction. The $y$-axis was the rotating axis with a constant angular velocity, $\Omega$, as shown in Fig.1b where $r$ is the radial vector from the rotating axis. With $r=\left(0,0,-r_{0}\right)$, it is assumed that $r_{0}$ is constant for the whole domain 


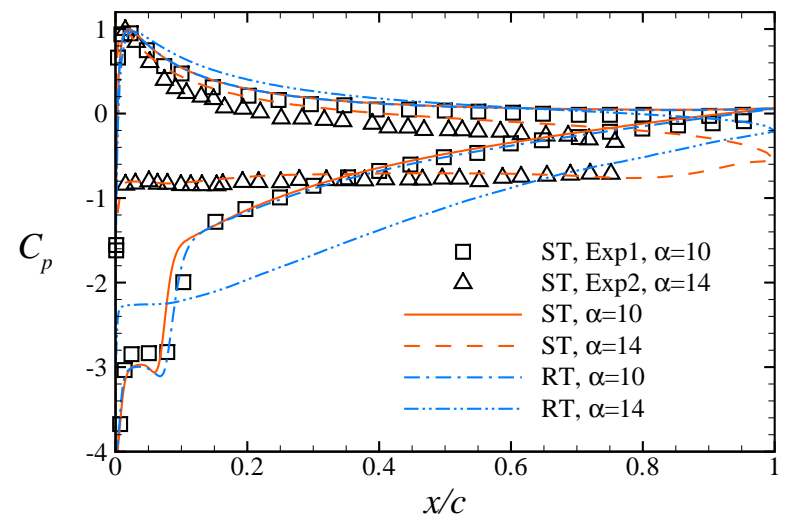

Fig. 2 Pressure coefficients from experiments (Exp1: [9], Exp2: [7]), stationary (ST) and rotating (RT) airfoil cases.

so that the source term on the right-hand side of the Navier-Stokes equations in Cartesian coordinates is

$$
f_{s}=-\left(2(\Omega \times U)+\Omega \times(\Omega \times r)+\left(\frac{u w}{|r|}, 0,-\frac{u u}{|r|}\right)^{T}\right) .
$$

Note that $f_{s}, \Omega, U$ are vectors. The number of grid points around the airfoil was increased by using a local refinement until the pressure and skin-friction were converged. The domain width was $L_{z}=0.25 \mathrm{c}$ and the total number of cells was about $21 \times 10^{6}$ with 942 grid points along the airfoil surface and 128 points in the spanwise direction near the airfoil. The first off-wall grid point was placed at $y_{1} \approx 7 \times 10^{-5} c$. Averaging started once the lift coefficient reached a statistically converged state and the averaging was done over about $8 T$, where $T=c / U_{\infty}$; the time step was $t / T=0.00015$.

Two different flow conditions, pre- (i.e. $\alpha=10^{\circ}$ ) and post- (i.e. $\alpha=14^{\circ}$ ) stall, were simulated as baseline cases and compared with experimental data [7, 9]. Pressure coefficients are shown in Fig.2. The peak pressure plateau, size of bubble and separation points both for the pre- and post-stall cases are predicted well compared with the reference data. Based on these stationary cases, the rotating blade was simulated. Rotation numbers $R o=\Omega c / U_{\infty}$ are 2.27 and 1.60 for pre- and post-stall cases respectively. When the flow is attached (e.g. at $\alpha=10^{\circ}$ in Fig.2), there is little difference between the flows over the stationary and rotating airfoils. This is because the spanwise flow due to the centrifugal force is weak which leads little Coriolis acceleration. When the flow is detached (i.e. at $\alpha=14^{\circ}$ ), however, the spanwise flow increases, leading to a strong and favourable pressure gradient in the chord-wise direction, generated by the Coriolis force.

The mechanism for rotational augmentation has been explained mainly in two ways. Firstly, the centrifugal force pushes the air to the tip and the mass depletion 

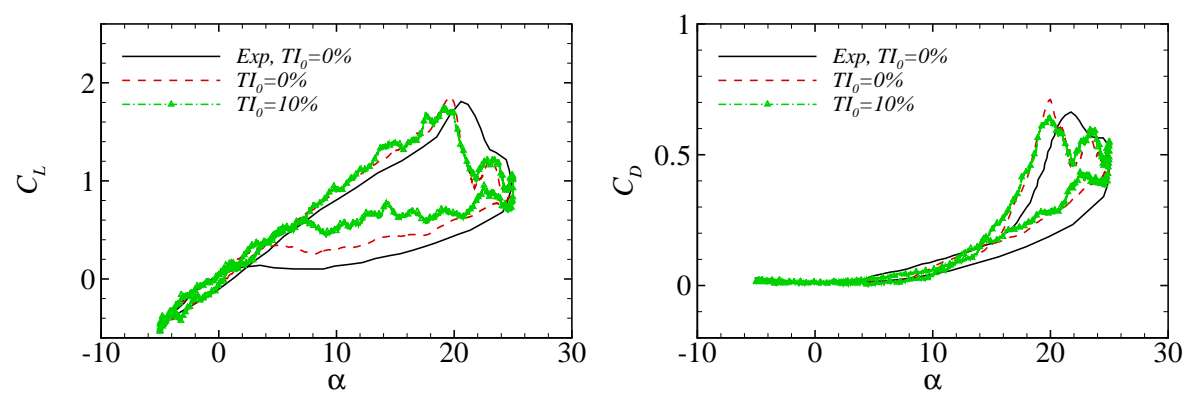

Fig. 3 The effect of freestream turbulence on the lift and drag coefficients. Note that the suffix " 0 ' indicates the input variables. Exp is taken from [7]

due to the radial flow thins the boundary layer thickness leading a low pressure on the suction side. Secondly, the radial flow to the blade tip provides Coriolis acceleration toward the trailing edge. This acceleration acts as favourable pressure gradient thus increases the lift.

Gross et al. [2] further scrutinized that the spanwise flow provides cross-flow instability which triggers early transition thus it delays separation and suppresses the boundary layer. They excluded the mass depletion effect by adopting the periodic boundary condition in the spanwise direction but did not discuss on the contribution of the Coriolis effect on rotational augmentation. In order to further specifically identify the problem, we excluded considering the mass depletion as in [2] and used a NACA 0012 airfoil rather than a S833 airfoil. This airfoil is a leading edge separation type and the transition point is very close to the leading edge. Thus it is expected that the effect of the early transition by the cross-flow instability would be minor for this airfoil. Therefore it is demonstrated that the Coriolis acceleration is a primary mechanism for rotational augmentation.

\section{Dynamic stall}

Dynamic stall is a phenomenon associated with an unsteady airfoil (or lifting surface) motion that presents large hysteresis on lift, drag and pitching moment while the incidence is beyond its static stall angle. For the dynamic stall study, a pitching airfoil was simulated by using a dynamic mesh [8]. The pitching motion was $\alpha(t)=10+15 \sin (\omega t)$. The reduced frequency, $k_{\text {red }}\left(=\frac{\omega c}{2 U_{\infty}}\right)$, was 0.05 where $\omega$ was the pitching frequency. This frequency is typical of operating conditions for wind turbines.

The effect of freestream turbulence over a static airfoil is significant [5]. The effect of freestream turbulence on the flow over a pitching airfoil is reported here. The divergence-free turbulence inflow condition was applied on a 2-D transverse plane placed at $7 c$ upstream from the leading edge. The turbulent intensity $(T I)$ was set to $10 \%$ and it naturally decays along the streamwise direction. The turbulent 

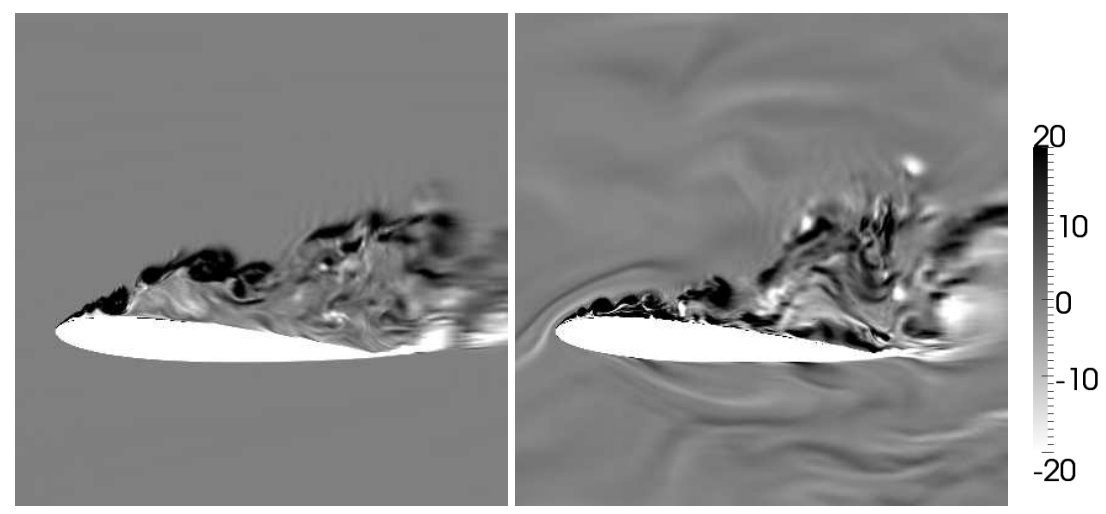

Fig. 4 Instantaneous spanwise (z) vorticity component at the mid-span at $\alpha=14.2 \downarrow$ with $T I_{0}=$ $0 \%$ (left) and with $T I_{0}=10 \%$ (right). The vorticity fields are normalized by $U_{\infty}$ and $c$.

intensity at the leading edge location was $T I=6.3 \%$, and kept nearly constant down to the trailing edge location when the airfoil was not placed in the domain.

Mesh convergence tests were carefully conducted with various resolutions and domain sizes. The optimal mesh was chosen and the number of grid points was $738 \times 205 \times 20$ in the circumferential, cross-flow and spanwise directions respectively. The first grid points from the airfoil were located at $y_{1} \approx 2 \times 10^{-4} c$. The domain width in the spanwise direction was set to $L_{z}=0.5$ and a symmetric boundary condition was applied in this direction.

Lift and drag coefficients for pitching airfoil cases are shown in Fig.3. The numerical results are taken from the second cycle and two cycles were used for the phase average. The calculated lift and drag hysteresis generally show good agreement with the experimental data with $T I_{0}=0 \%$. The maximum lift occurs at slightly lower incidence and the hysteresis loop is smaller for the calculation than that in the experiment.

Generally freestream turbulence does not significantly change the drag and moment (not shown) hysteresis at the given conditions as shown in Fig. 3. It only slightly reduces the maximum drag coefficient, which might because the freestream turbulence suppress the separation flows. The angles for the maximum lift and drag are nearly the same as those for case $T I_{0}=0 \%$. But it is evident that freestream turbulence does have an impact on the lift during the downstroke. The lift increases for the turbulence inflow case compared with that for the laminar inflow case and the increment is about $\Delta C_{L} \approx 0.2$. This is again because the separated flow is suppressed by freestream turbulence. Amandolèse and Széchényi [1] measured the upstream turbulence effects on the flow over a pitching airfoil. Similarities can be found from their works although the setting parameters are not the same.

Fig. 4 shows snapshots of the effect of freestream turbulence on the pitching airfoil during the downstroke. The separated flows are significantly suppressed by the freestream turbulence. The interactions are also found evidently in other incidence 
during the downstroke. These interactions lead a decrease of the separated region and an increase of the lift during the downstroke.

\section{Conclusion}

This paper addresses three challenging points in LES/DNS approaches for wind turbine flows, namely modelling rotation augmentation, using dynamic mesh and inflow generation. The required massive computational resources (i.e. simulations of a few cycles of pitching motion) make these tasks even more difficult in practice. Up to our knowledge, this is the first attempt to tackle these problems. Our numerical work is novel in terms of identifying the mechanism which is crucial in understanding these flows. We exclusively identify that the effect of the Coriolis acceleration is a primary factor on rotational augmentation for airfoils with a leading edge separation. The effect of freestream turbulence on dynamic stall is investigated using our recently developed efficient inflow generator, and is found not small which confirms the experiments in the literature. In addition, the LES capability has been demonstrated for highly separated and strong 3-D flows, e.g. the dynamic stall.

Acknowledgements YK acknowledges provision of a Ph.D studentship from FEE, University of Southampton. All the computations were performed on supercomputers IRIDIS3 and HECToR.

\section{References}

1. Amandolèse, $X$. and Széchényl, E.: Experimental study of the effect of turbulence on a section model blade oscillating in stall. Wind Energy. 7, 267-282 (2004)

2. Gross, A., Fasel, H.F., Friederich, T., Kloker, M.J.: Numerical investigation of rotational augmentation for S822 wind turbine airfoil. Wind Energy. 15, 983-1007 (2012)

3. Inagaki, M., Kondoh, T., Nagano, Y.: A mixed-time-scale SGS model with fixed modelparameters for practical LES. J. Fluids Eng. 127, 1-13 (2005)

4. Jasak, H.: Error analysis and estimation for the finite volume method with application to fluid flows. PhD thesis. Imperial College of Science, Technology and Medicine (1996)

5. Kim, Y., Castro, I.P., Xie, Z.T.: Divergence-free turbulence inflow conditions for large-eddy simulations of flows around an airfoil. UK WES conference (2012)

6. Kim, Y., Castro, I.P., Xie, Z.T.: Divergence-free turbulence inflow conditions for large-eddy simulations with incompressible flow solvers. Compt. Fluids. In press (2013)

7. Lee, T. Gerontakos, P.: Investigation of flow over an oscillating airfoil. J. Fluid Mech. 512, 313-341 (2004)

8. OpenFOAM (R): User guide v1.7.1. OpenFOAM (2010)

9. Rinoie, K., Takemura, N.: Oscillating behaviour of laminar separation bubble formed on an aerofoil near stall. The Aeronaut. J. 153-163 (2004)

10. Schreck, S., Robinson, M.: Rotational augmentation of horizontal axis wind turbine blade aerodynamic response. Wind Energy. 5, 133-150 (2002)

11. Simms, D., Schreck, S., Hand, M., Fingersh, L.J.: NREL Unsteady aerodynamics experiment in the NASA-Ames wind tunnel: A comparison of prediction to measurements. NREL/TP500-29494, NREL (2001) 